\title{
HTEE: AN HMAC BASED TAMPER EVIDENT ENCRYPTION
}

\author{
Bradley Baker, C. Edward Chow $\dagger$ \\ Department of Computer Science, University of Colorado at Colorado Springs \\ 1420 Austin Bluffs Parkway, Colorado Springs, CO 80918, USA \\ bbaker@uccs.edu,chow@cs.uccs.edu
}

Keywords: Encryption, Integrity, Confidentiality, HMAC, Tamper Detection, Hash

Abstract: This paper presents a HMAC based Temper Evident Encryption (HTEE) technique for providing confidentiality and integrity of numeric data in a database environment through an encryption scheme based on the keyed Hash Message Authentication Code (HMAC) function. The encryption scheme implemented in this project extends and improves an existing HMAC based encryption scheme. The result is a symmetric encryption process which detects unauthorized updates to ciphertext data, verifies integrity and provides confidentiality. This encryption scheme provides an alternative to standard approaches that offer confidentiality and integrity of data such as combining the Advanced Encryption Standard (AES) algorithm with a hash digest. The purpose of the scheme is to provide a straightforward and efficient encryption that supports data integrity, to investigate the use of HMAC for reversible encryption and key transformation, and to improve upon an existing method.

\section{INTRODUCTION}

Databases are used to store a wide variety of sensitive data ranging from personally identifiable information to financial records and other critical applications. The volume and importance of sensitive data stored and processed electronically is constantly growing, and this data must be protected from unauthorized disclosure or modification. Confidentiality and integrity of this sensitive data must be maintained for legal or fiscal reasons (Pavlou and Snodgrass, 2008), (Kher and Kim, 2005). Due to the wide range of problem domains, a variety of solutions are of interest to suit particular situations (Sivathanu et al., 2005).

This paper provides confidentiality and tamper detection in a database environment. Existing work supports tamper detection and integrity for database systems using techniques such as access control, auditing and other methods. Additional related work includes forensic analysis of database tampering (Pavlou and Snodgrass, 2008). Some techniques

$\dagger$ : This research work was supported in part by two NISSSC AFOSR grant awards under numbers FA9550-06-1-0477 and FA9550-04-1-0239. apply encryption and authentication in parallel to provide confidentiality and integrity (Torres et al., 2006a), (Torres et al., 2006b). Unlike these techniques, this paper uses an encryption scheme based on the keyed Hash Message Authentication Code (HMAC) (Bellare et al., 1996) (NIST, 2002) for confidentiality and integrity. Existing work uses HMAC for integrity but it is not typically used for confidentiality. An exception is presented by Lee et al. (2007), which investigates HMAC as an encryption function.

The encryption scheme used for this paper offers tamper detection and confidentiality directly in the encrypted data field rather than externally or at the system level. Cryptography provides standard algorithms that also support confidentiality and integrity in the encrypted data field, including symmetric and asymmetric encryption algorithms for confidentiality and hash digest or signature algorithms for integrity. Combining these solutions can require detailed processing by the end user and may not be ideal for all problem domains.

\subsection{Project Overview}


In a database record sensitive data is paired with information that uniquely identifies the record such as primary key or hash digest. Each row in a database table contains a combination of uniquely identifying information and sensitive data, and this relationship must be preserved from encryption through decryption. The relationship can be tampered with while data is encrypted, when this occurs the integrity of the data is lost.

Typically encryption algorithms such as the Advanced Encryption Standard (AES) provide confidentiality but don't provide integrity and hash digest algorithms such as Secure Hash Algorithm (SHA) provide integrity without confidentiality (Forouzan, 2008). Traditional methods to obtain both confidentiality and integrity involve combining encryption and digest algorithms. Message authentication codes such as HMAC provide an alternative to traditional hash digests where the digest is protected from unauthorized update with a secret key.

This paper presents a HMAC based encryption scheme that provides confidentiality and tamper detection for positive integer data. This scheme is an improvement in efficiency and tamper detection to the HMAC integer encryption concept presented in (Lee et al. 2007). The scheme is implemented in the PostgreSQL database environment (PostgreSQL, 2009), and the developed process is named "HMAC based Tamper Evident Encryption”, referred to as HTEE in this paper. This process is simpler to use than the standard AES with SHA solution, and more efficient for encryption. However this process is slower on decryption than AES with SHA, and the security of this scheme is dependent on the security of the underlying hash function.

The HTEE scheme is a symmetric encryption process that relies on a secret key and processes positive integer values. The integer plaintext values are decomposed into components, or buckets, using modulus arithmetic. The buckets have a fixed size of 1,000 , so integer values are decomposed into the value of the ones, thousands, millions, etc. places. The plaintext buckets are encrypted using the HMAC function, where the hash digest represents the ciphertext. The secret key is modified for each plaintext value and each bucket value using a specific transformation process resulting in a different key for every HMAC operation. The key transformation process is based on a unique value related to the sensitive data, such as a database primary key. A primary goal of the HTEE process is the detection of unauthorized updates or tampering with ciphertext data, particularly when ciphertext values are interchanged. The key transformation process ensures ciphertext values can’t be changed without detection.

The decryption process is similar to the encryption process and uses the same key transformation sequence. Because the HMAC function produces a one-way hash digest, it is not trivial to reverse the operation. In order to find the correct plaintext for each bucket's digest value a search is performed across all 1,000 possible bucket values, calculating the HMAC digest of each until a match is found. The search is repeated for all buckets and the modulus decomposition is reversed to obtain the plaintext value. Any unauthorized updates to ciphertext data are detected in the decryption step by a failure to find a matching HMAC digest.

\section{BACKGROUND}

\subsection{Hash Message Authentication Code}

HMAC is a symmetric process that uses a secret key and a hash algorithm such as SHA to generate a message authentication code, or digest. This authentication code securely provides data integrity and authenticity because the secret key is required to reproduce the code. Digests for normal hash functions can be reproduced with no such constraint. HMAC can protect against man-in-the-middle attacks on the message, but it is not designed to encrypt the message itself. The HMAC function was published by Bellare et al. (1996), which includes analysis and a proof of the function's security, and it is standardized in FIPS PUB 198 (NIST, 2002). Any hash algorithm can be used with HMAC including MD5, SHA-1, SHA-256, etc.

The output of HMAC is a binary authentication code equal in length to the hash function digest. The security of HMAC is directly related to the underlying hash function used, so it is weaker with MD5 and stronger with SHA-512. Forgery and key recovery attacks threaten HMAC, but typically require a large number of message/digest pairs for analysis. The HMAC functions used in the implementation of the HTEE scheme are based on the SHA-1 hash algorithm. The use of HMACSHA1 specifies some data sizes that are important in the HTEE implementation such as a 64 byte key size 20 byte digest output size.

\subsection{HMAC Integer Encryption}


The HTEE algorithm is based on an original HMAC encryption scheme presented by Lee et al. (2007), and provides several improvements. A detailed analysis and discussion of this original scheme is available in (Baker, 2009a). The original scheme uses integer decomposition, HMAC for encryption, and decryption with exhaustive search. Because the original scheme does not combine related data with the plaintext data it cannot be used for tamper detection.

The original encryption scheme takes a positive integer input as plaintext, and computes the remainder of the plaintext and a predefined bucket size. After calculating the remainder a bucket ID is found as the quotient of division between plaintext and bucket size.

Encryption uses a secret key, a seed value, the plaintext bucket ID and the remainder. The encrypted bucket ID is found by calculating the HMAC function recursively $\mathrm{N}$ times, where $\mathrm{N}$ is equal to the bucket ID. On the first iteration, the secret key and a predefined seed value are input into HMAC. For successive iterations, the output of the previous HMAC is used as input into the next iteration with the secret key. The bucket ID is not directly encrypted, but the execution of recursive HMAC is based on the value of the bucket ID.

The encrypted value for the remainder is found in a similar operation differing only in the secret key. When encrypting the remainder value the corresponding bucket ID is appended to the beginning of the secret key to form a new key. The recursive HMAC operation is the same using the new key. Beginning with the seed, the digest is calculated $\mathrm{N}$ times where $\mathrm{N}$ is equal to the value of the remainder.

Decryption uses an inverse transformation that must search through potential bucket ID and remainder values. The maximum bucket ID must be defined to constrain the search process. The first step for the decryption transformation is finding the bucket ID of the ciphertext data. The same seed and key value from encryption are used in the HMAC operation, and this operation is executed $\mathrm{N}$ times for the number of possible buckets. Each HMAC digest is compared against the encrypted bucket ID for a match. If a match is found, the bucket ID plaintext is equal to the number of iterations executed.

A similar search is made for the remainder value using a new key constructed by appending the decrypted bucket ID to the beginning of the secret key. Once the plaintext bucket ID and remainder values are known, the modulus decomposition is reversed to generate the original plaintext from the decrypted bucket ID and remainder.

Issues identified with the original scheme include the problem that two buckets decrease efficiency for large integer values, the key transformation only occurs on the remainder value rather than the bucket ID, and the highly recursive use of HMAC is inefficient (Baker, 2009a).

\section{DESIGN}

The HTEE process is similar to the original HMAC encryption scheme in that positive integer values are processed, these values are decomposed into components, also called buckets, and the bucket values are processed through HMAC for encryption. The combination of HMAC output for all bucket values creates the ciphertext. The decryption step calculates the HMAC digest for all possible bucket values, where a match between calculated digest and ciphertext data indicates the correct plaintext result. HTEE uses multiple smaller buckets to reduce decryption search ranges, and it adds a key transformation process that ensures each bucket of each plaintext uses a different encryption key. The key transformation process ensures tamper detection.

\subsection{Plaintext Decomposition}

The first step of the encryption process is decomposition of the integer plaintext input. In the HTEE scheme, the integer plaintext value is decomposed into multiple buckets of size 1,000 to improve search efficiency. The number of buckets used for a given plaintext is calculated with:

$$
\text { floor }\left(\log _{1000}(\text { Plaintext })\right)+1
$$

Because each bucket produces one HMAC digest value, larger plaintext values will produce a larger ciphertext. In order to avoid leaking information about the plaintext's order of magnitude, a domain specific maximum number of buckets are defined and small plaintext values are padded. Using more buckets of smaller sizes allows the decryption operation to be more efficient because a smaller number of HMAC searches must be performed.

Additional improvements to performance can be achieved if fewer buckets are needed in a problem domain, such as storing nine digit values versus sixteen digit values. 


\subsection{Key Transformation}

The second step of the encryption process is key transformation, which prepares distinct secret keys for the encryption of each bucket value. The HTEE scheme improves the original process and adds tamper detection by defining two key transformation functions, an element transformation and a bucket transformation. The element key transformation creates a new secret key for each plaintext value processed. This transformation is seeded with information relating the plaintext data to its environment, providing tamper detection. The bucket key transformation produces a new secret key used on each decomposed bucket value of a given plaintext. The bucket key is the effective encryption key because only decomposed bucket values are encrypted. The method of key transformation used for bucket values also contributes to tamper detection because it is a continuation of the element key process. Both the bucket and element transformations use the HMAC function to generate new secret key data. For its use here as a key transformation function, HMAC is considered a pseudo-random value generator. Research supports HMAC as a pseudo-random function, as discussed in (Bellare et al. 1996), (Bellare 2006), (Canetti 2007), (Kim et al. 2006). The key transformation functions used for HTEE provide a critical security feature that makes analysis of the ciphertext output more difficult.

\subsection{Element Key Transformation}

The HTEE scheme transforms the element key based on a unique value. This process constructs an element key using the original secret key and uniquely identifiable data related to the plaintext value. Usually the unique value is the primary key of the database record, but any data unique to the plaintext can be used. The hash digest of the unique value is found with the SHA-1 algorithm, and used as input into the HMAC function alongside the original secret key. The output of this HMAC operation is used for the first 20 bytes of the element key, and it is used as input into another HMAC operation with the original secret key. The output of the second operation is used for the second 20 bytes of the element key, and it is processed through HMAC again. This process repeats until four recursive HMAC operations are executed, outputting 80 bytes of key data. The output is then truncated to 64 bytes, producing the element key. This process is depicted graphically in Figure 1.
An attacker cannot reproduce the key if given the unique value, because the process is secured with the HMAC function and secret key. The key transformation process is important for HTEE tamper detection because it incorporates information related to the plaintext value with the encryption of the value. The result is that decryption of the ciphertext is dependent on the unique value, and any changes between ciphertext and unique value can be detected.

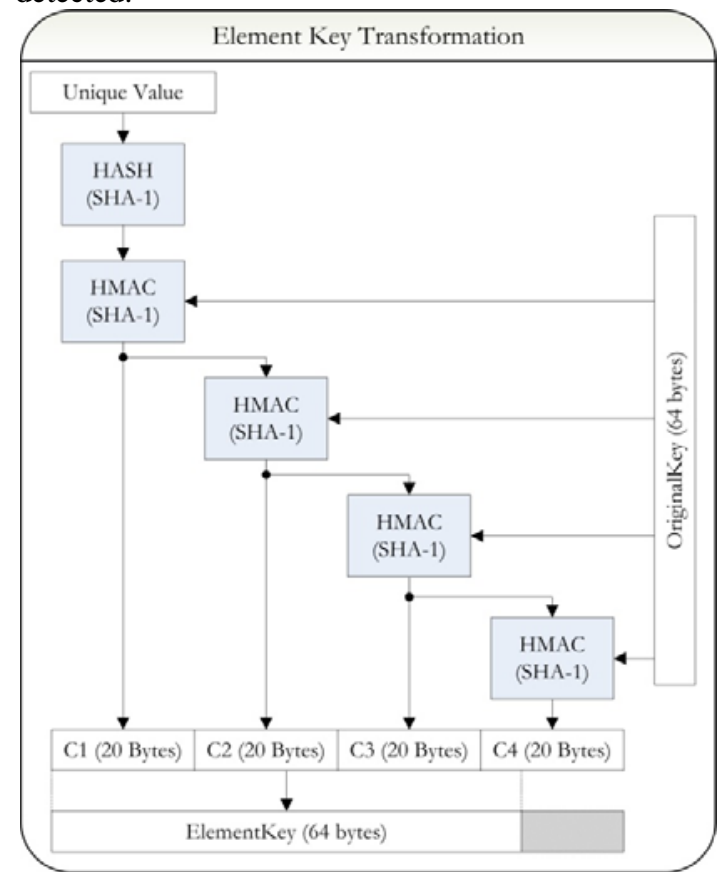

Figure 1 - Element key transformation.

\subsection{Bucket Key Transform}

The second key transformation function used by HTEE is the bucket key transformation. The HTEE process uses a different key for each bucket's HMAC function so that buckets with equal values do not have equal digests. The bucket key transformation is iterative, and 20 bytes of the bucket key are replaced for each bucket processed in a plaintext. The first bucket key is equal to the element key generated for the plaintext value. Each succeeding bucket key is generated by processing the bucket's HMAC encryption ciphertext through HMAC again with the original secret key. The result of this HMAC operation is appended to the beginning of the bucket key, and the result is truncated to 64 bytes resulting in the succeeding bucket key. 


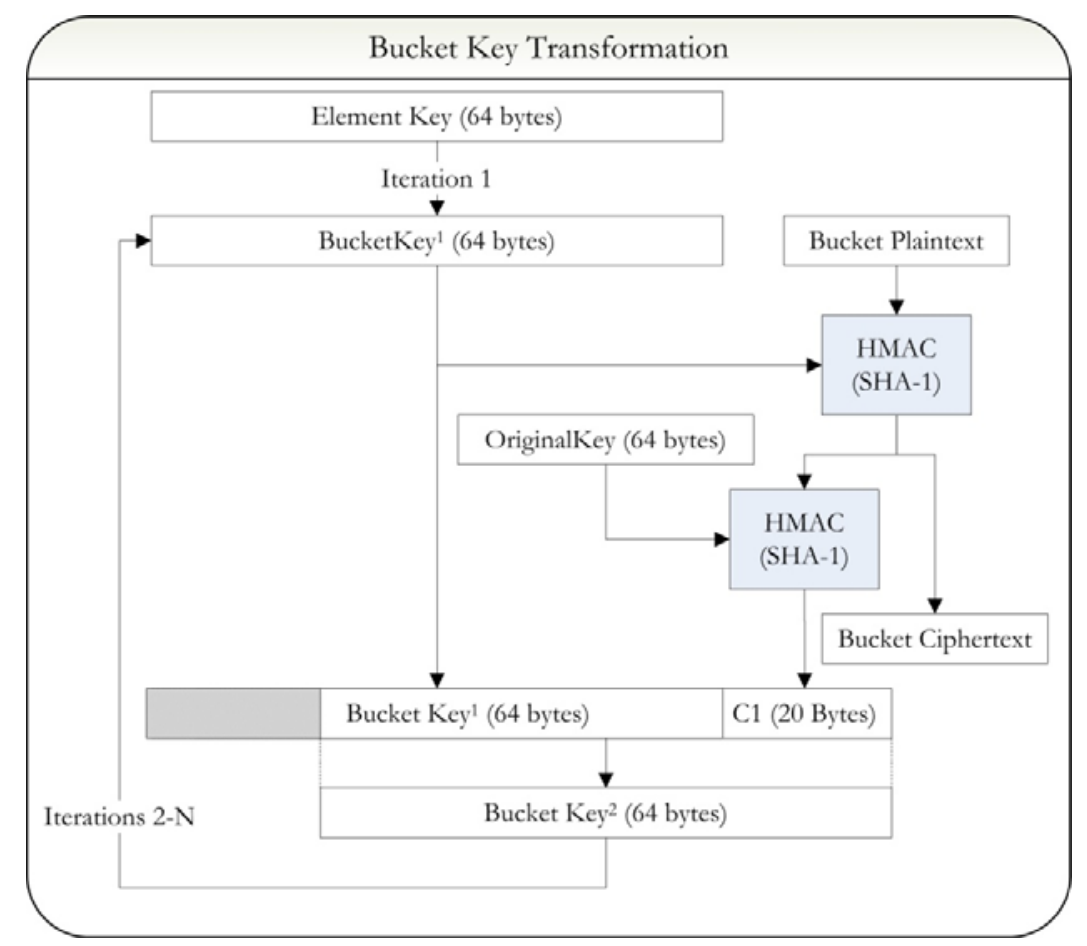

Figure 2 - Bucket key transformation and encryption.

The bucket key transformation is summarized graphically in Figure 2. The function presented in Figure 2 depicts both the calculation of the bucket ciphertext as well as the transformation of the bucket key. The bucket key transformation makes encryption keys dependent on both the unique value used to generate the element key, and the order of processing for the bucket values. The combination of element and bucket key transformations produces distinct keys for each plaintext bucket value provided that differing unique values are input. The only cases when the key generation process will not result in distinct keys are for hash collisions of the unique value data, which are extremely rare cases.

\subsection{Encryption}

The encryption step of the process calculates the HMAC digest using the key and plaintext values for each bucket. The digests are concatenated to form the ciphertext output.

The HTEE encryption operation is a very efficient process regarding computation time, because the HMAC function is executed a small number of times. For example, when processing a plaintext value using four buckets, HMAC will be invoked twelve times. However, the decryption process for HTEE presents a performance challenge due to the need for exhaustive searching across possible plaintext values. In the example of a four bucket plaintext, HMAC could be executed up to 4,008 times.

\subsection{Decryption}

The HTEE decryption operation is similar to the encryption operation, particularly with the key transformation functions. The same progression of element keys and bucket keys is calculated, except these keys are used for a search across all plaintext bucket values. The first step in the decryption process is splitting the concatenated ciphertext string into individual bucket digests. Then the key transformation process is used with the unique value data (which cannot be encrypted) to find the same bucket key values used during encryption. The process then iterates through all possible bucket plaintext values, 0 through 999, calculating the HMAC digest for each one with the bucket key. The intermediate digest is compared with the stored bucket digest, if the values match then the current iteration is the bucket's plaintext value. If no records from 0 through 999 match the bucket digest, then some corruption or tampering of the ciphertext has 
occurred. This step is the critical tamper detection operation for HMAC; the absence of a correct decryption match indicates that the ciphertext data or unique value has changed since encryption. Once all bucket plaintext values are identified, the modulus decomposition is reversed.

\section{ANALYSIS}

\subsection{HMAC security}

The security of the HTEE scheme is primarily based on the security of the HMAC function, because HMAC is used for both key transformation and encryption. Existing work has established that the security or cryptographic strength of the HMAC function is directly related to the security of the underlying hash function on which is it based (Bellare et al. 1996), (Bellare 2005), (Bellare 2006). Although recent findings on collision attacks have invalidated the use of the MD5 hash algorithm and decreased confidence in the SHA-1 algorithm, these attacks have limited impact on HMAC security. HMAC is proven to be secure provided that the hash compression operation is a pseudo-random function (Bellare 2006), (Contini et al. 2006). In addition the secret key reduces the effect of collision based attacks on the HMAC function (Bellare 2005), (Bellare 2006), (Kim et al. 2006).

While the strength of HMAC security is based on the compression operation of the underlying hash function, the measure of security is the difficulty to produce a forgery of the authentication code. There are several methods researched to produce forgeries in the HMAC function, the primary being the birthday attack. Although collisions of the underlying hash function are not a concern for the HMAC, it is still the case the HMAC output is a digest of a message and secret key input, and it can produce its own collisions. It is possible for an attacker to observe two different messages that have the same digest output. The probability of this occurrence is controlled by the birthday paradox, where a HMAC collision becomes probable after $2^{\mathrm{n} / 2}$ message pairs are observed, where $n$ is the number of bits in the output digest (Bellare 2005 ), (Kim et al. 2006). A HMAC-SHA1 function would be susceptible to a forgery based on the birthday paradox after $2^{80}$ message pairs are observed. When attacking HMAC with the birthday paradox, the attacker relies on a legitimate user to generate all $2^{80}$ digests. Also the effect of a birthday attack is a forgery, and does not yield the secret key so impact is limited.

Full key recovery attacks are another threat to the HMAC function. These attacks still appear infeasible, although some methods have efficiency improvements (Fouque et al. 2007), (Contini et al. 2006), (Sasaki 2009). These methods have an underlying requirement of a very large number of HMAC message/authentication code pairs for analysis, more than are required for the birthday attack.

\subsection{HTEE security}

In the context of the HTEE scheme, the HMAC operation is secure considering typical birthday and key recovery attacks. In an environment with $2^{40}$ records and six buckets of HMAC digest data for each record, this is not close enough to the number of messages required to perform key retrieval or birthday attacks if HMAC-SHA1 is used (Bellare et al. 1996), (Fouque et al. 2007), (Contini et al. 2006).

An additional consideration for security of the HTEE scheme includes the input of unique value and plaintext value as messages for the HMAC function. The data ranges for unique value can vary widely according to the problem domain, and the plaintext value will always have a small range due to the HTEE bucket decomposition limiting values to integers (0-999). The key transformation process provides a layer of protection for small values because any analysis of the ciphertext data will be challenged with constantly varying keys. However, the key transformation process begins with the unique value input which is known to the attacker since it cannot be encrypted in the database. A likely method for an attacker to pursue is attacking the key transformation function using the unencrypted unique values. The natural variation of the unique value is masked by the hash and recursive HMAC functions in the element key transformation.

Considering the use of HMAC as a pseudorandom function, the variation in key values through the transformation process should be unpredictable. This is expected even if the unique value size is small, due to the pseudo-random feature of the underlying hash compression function. Additional data could be provided for the unique related value, thus expanding it beyond the range of small input values.

The structure of the HTEE scheme provides additional protection by obscuring internal values in a similar way to the inner and outer hash operations of the HMAC function. Consider that the attacker 
knows two values: the ciphertext output from HTEE, and the unique value input. The HTEE function can be written in a short format as:

$$
\operatorname{HTEE}(P, K, U)=\operatorname{HMAC}\left(P, f_{K}(K, U)\right)
$$

Where $\mathrm{P}$ is the plaintext value, $\mathrm{K}$ is the original secret key, $U$ is the unique value, and $f_{K}$ is the key transformation function. The $f_{K}$ function is $a$ combination of several HMAC steps as described previously, and produces intermediate keys. It is difficult for the attacker to generate the intermediate key used with a plaintext value, based on the analysis of HMAC key recovery attacks. It is also difficult for the attacker to identify the secret key using the unique input message because the result of function $\mathrm{f}_{\mathrm{K}}$ is not known.

\subsection{HTEE Tamper Detection}

Tamper detection is an important feature of the HTEE scheme and can be defined as the failure in data integrity between the ciphertext and the remainder of the database record. The data integrity relationship can be defined at a minimum as the record's primary key and the plaintext/ciphertext value.

An attacker can try to modify the data record in three ways: Case 1) Make a random change to ciphertext, Case 2) Interchange two ciphertext values and Case 3) Make a change to the unique value. The tamper detection feature of HTEE will detect each of these changes through the decryption viability test. If the modifications in Cases 1 or 2 were used, the unique value would be unchanged and the key transformation sequence for decryption would be identical to the encryption operation. Each step in the decryption search would iterate through possible plaintext values, but none of the HMAC digests would match the stored value. The probability of a false positive would be extremely small, approximately $3.42 \times 10^{-43}$, based on the birthday attack with 1,000 values (Forouzan 2008). This result is obtained with the formula:

$$
\mathrm{P}=1-\mathrm{e}^{\left(-\mathrm{k}^{\wedge} 2 / 2 \mathrm{~N}\right)}
$$

Where $\mathrm{k}$ is the sample size, equal to 1,000 and $\mathrm{N}$ is the number of possible values, equal to $2^{160}$ for SHA-1. If the modification in Case 3 was used, the key transformation sequence would be changed resulting in a similarly improbable collision. The new key transformation and a value between (0-999) would have to collide with the original transformed key and a value between (0-999).

\section{IMPLEMENTATION}

The HTEE scheme was implemented to validate the designed algorithm, evaluate performance, and provide a tool that could be used for future applications. The implementation is an add-on for the PostgreSQL database management system and provides encryption and tamper detection features.

The implementation uses the HMAC operation with SHA-1 as underlying hash function and for the element key transformation. The use of HMACSHA1 specifies several parameter sizes that are important during implementation including the key size of 64 bytes and the digest size as a multiple of 20 bytes per bucket. The bucket size used for the implementation is 1,000 , which breaks numbers into buckets by order of magnitude such as millions, billions, etc.

Each bucket value is up to three plaintext digits (values 0-999) which are encrypted into 28 base64 encoded characters. A six bucket HTEE ciphertext would require 168 bytes of text data. This is a ninefold increase in storage space when the plaintext is stored as a text string. However, the equivalent AES ciphertext requires 116 bytes of base64 text data in PostgreSQL, so HTEE is only a $44 \%$ increase over the AES requirement. The large increase in storage space is one of the costs of using the more efficient small bucket solution employed by HTEE. The other primary cost is decryption processing time.

\subsection{Testing Summary}

Several tests were performed on HTEE including comparisons to AES based techniques. Three encryption techniques were tested in a PostgreSQL database system: 1) Raw AES encryption, 2) AES encryption with unique value data and 3) the HTEE encryption scheme. Method 1, the raw AES encryption scheme, is straightforward and uses AES with a secret key value. This method can detect random changes to ciphertext data, but it cannot detect other tampering. Method 2, using AES encryption with unique value data is a solution that adds tamper detection to the raw AES encryption. The approach used for AES tamper detection includes concatenating the unique value data with the plaintext data, and encrypting the combined string. On decryption, the unique value is separated from the plaintext, and the plaintext is recovered. If 
the decrypted unique value differs from the current unique value, the data was tampered with. The HTEE encryption scheme used the primary key as unique value and managed tamper detection internally.

The testing process used six datasets, each composed of 20,000 randomly generated integers. The datasets were each configured with a different number of buckets, so one dataset had values between 0 and 999 (one bucket), another dataset had values between 1,000 and 999,000 (two buckets), etc. up to the six buckets or 18 digits. Performance was timed for the encryption, decryption and tamper detection operations. The tamper detection dataset was built by interchanging half of the ciphertext records.

\subsection{Testing Results}

Performance results from testing are summarized in Table 1. The average performance times demonstrate the trade-off in efficiency between the HTEE scheme and AES based schemes. The encryption operation for AES with tamper detection was about 4.5 times slower than the encryption operation for HTEE. Conversely, the decryption operation for HTEE was about 4.1 times slower than the decryption operation for AES.

Table 1 - Average performance across bucket sizes.

\begin{tabular}{|l|l|l|}
\hline \multicolumn{3}{|c|}{$\begin{array}{c}\text { Average Performance } \\
\text { (time in seconds) }\end{array}$} \\
\hline Encrypt Method & Mode & Time \\
\hline Original AES & encrypt & 18.1 \\
Original AES & decrypt & 15.3 \\
Original AES & tamper & 18.3 \\
\hline Tamper Detect AES & encrypt & 15.8 \\
Tamper Detect AES & decrypt & 18.2 \\
Tamper Detect AES & tamper & 17.8 \\
\hline HTEE & encrypt & 3.5 \\
HTEE & decrypt & 75.4 \\
HTEE & tamper & 58.8 \\
\hline
\end{tabular}

Performance of HTEE varies according to the bucket size used. The number of buckets processed affected HTEE encryption marginally, but more buckets decreased performance of decryption and tamper detection greatly. This was due to the exhaustive search required for decryption, where processing time increases with number of buckets.
Efficiency improved for tampered datasets because the process could identify the tampering early in processing. The AES methods provide consistent performance for encryption and decryption - near seventeen seconds for each run regardless of bucket size. The HTEE scheme provides consistent fast performance for encryption at less than five seconds per run, but the processing time for decryption increases to over two minutes depending on the number of buckets processed.

\subsection{Performance Analysis}

The performance results from testing indicate a fourfold decrease in encryption time and four-fold increase in decryption time over AES. This would be a reasonable trade-off for some encryption heavy domains. The HTEE scheme also shows a performance improvement over the original HMAC encryption scheme (Lee et al. 2007) based on the algorithmic structure of the methods. The performance of the two schemes is generalized based on the number of HMAC operations required for encryption and decryption. The complexity of the HTEE algorithm can be summarized as approximately:

$$
\begin{gathered}
2 * \log _{1000}(\mathrm{n}) \\
1001 * \log _{1000}(\mathrm{n})
\end{gathered}
$$

Where (4) is the encryption complexity because of the encryption and key transformation HMAC functions, and (5) is the decryption complexity for the exhaustive search and key transformation.

These performance expectations are compared against the original HMAC encryption scheme. Based on the analysis of the original scheme presented in (Baker, 2009a), the encryption and decryption operations are equal in efficiency if processing a single plaintext value. For large numbers the complexity can be summarized as approximately:

$$
2 * n^{0.5}
$$

For both encryption and decryption because of the larger bucket sizes, ideally set to the square root of the maximum plaintext value. HTEE has a constant relative complexity, and the original scheme has polynomial performance.

Performance testing verifies the improvement in processing time with the HTEE scheme over the original HMAC encryption method. As presented in (Baker, 2009a), a test of the original scheme with 
2,000 integer values took 2 minutes and decryption took 3 minutes. These results are much slower than the HTEE performance times seen with all of the 20,000 integer datasets, represented by the average in Table 1.

\section{CONCLUSION}

The HTEE scheme provides a framework for tamper detection and encryption of integers in a database environment that can be useful in some applications. Benefits to the approach include the simplicity of a single-column confidentiality and integrity solution, trustworthy tamper detection based on a hash function, and efficient encryption speed. Drawbacks to the approach include inefficient decryption and increased volume of ciphertext.

The security analysis shows that the cryptographic strength of HTEE is based on the HMAC function and in turn the underlying hash function, SHA-1. Recent work suggests that HMAC is not affected by collision attacks against SHA-1 (Bellare, 2005). (Bellare, 2006). Key recovery attacks are a threat to the HTEE scheme but these are still considered infeasible, and require a very large number of valid HMAC authentication codes (Fouque et al. 2007), (Contini et al. 2006 ). Until a complete mathematical proof is generated, HTEE is considered not as secure as the AES encryption standard, and applications bound by regulatory requirements should continue to use AES methods.

The HTEE scheme is distinguished by plaintext decomposition into multiple buckets and secret key transformation functions. The multiple bucket solution makes decryption feasible for large integers, and key transformation functions increase security through layering and provide tamper detection through unique related values. The scheme can detect changes between a stored ciphertext value and other data related to it such as a record's primary key or hash digest value. The tamper detection feature is only provided on decryption, in order to be alerted to database tampering, the records must be decrypted.

The performance of the HTEE scheme is faster on encryption than AES, but slower on decryption. The differences are a factor of four in each case. For large numbers, the HTEE scheme is several orders of magnitude faster than the HMAC based encryption scheme it is based on. The HTEE scheme produces $44 \%$ more ciphertext data than an equivalent AES encryption scheme.
Applications for the HTEE scheme include areas where integer data is used, fast encryption speed is desired, slow decryption speed is not a significant concern, and tamper detection is needed. An example of this would be auditing systems or the archival of financial transactions. In these cases, a large number of records can be created on a daily basis, but the records might be infrequently referenced in the future. The HTEE method can support regular insertions into archive tables as opposed to a block encryption method that would require re-encryption of the entire data column. In a database that is write-only, or has little read access of encrypted records, HTEE can provide efficient tamper evident encryption as a supplementary protection for the database system. The full paper and project materials are presented in (Baker, 2009b)

\subsection{Future Work}

Some opportunities for future work related to the HTEE scheme include support for expanded plaintext values and a rigorous security proof. The HTEE scheme improved the original HMAC encryption concept to make encryption of larger integers (up to $9 \times 10^{17}$ ) feasible. However, the scheme is still limited to positive integer values because there is no way to encode negative or floating point values. A future improvement to the method could be a mechanism to process negative numbers, floating point numbers, and potentially ASCII-encoded text data.

This paper presented a conceptual argument for HTEE security based on existing work for HMAC security and key recovery. Based on the designed structure of HTEE, this provides a reasonable assurance of cryptographic strength because HMAC is the underlying function used, and it is widely considered to be a secure process. The security of HTEE is based on the HMAC function as a pseudorandom generator, both for key transformation and encryption. Future work can present a proof of the security for HTEE, which should focus on the random-generation capability of HMAC with the unique values used in the key transformation process.

\section{REFERENCES}

Brad Baker, 2009a "Analysis of an HMAC Based Database Encryption Scheme," UCCS Summer 2009 Independent study July. 2009 
URI: http://cs.uccs.edu/ gsc/pub/master/bbaker/doc/fi nal paper bbaker cs592.doc

Brad Baker, 2009b “Tamper Evident Encryption of Integers using keyed Hash Message Authentication Code" Project materials and documentation.

December 2009

URI = http://cs.uccs.edu/ gsc/pub/master/bbaker/

Forouzan, Behrouz A. 2008. Cryptography and Network Security. McGraw Hill higher Education. ISBN 978-007-287022-0

Mihir Bellare; Ran Canetti; Hugo Krawczyk; "Keying Hash Functions for Message Authentication”, IACR Crypto 1996

URI:

http://cseweb.ucsd.edu/users/mihir/papers/kmd5.pdf

Mihir Bellare, “Attacks on SHA-1,” 2005

URI:

http://www.openauthentication.org/pdfs/Attacks\%200 n\%20SHA-1.pdf

Mihir Bellare, "New Proofs for NMAC and HMAC: Security without Collision-Resistance," IACR Crypto 2006

URI: http://eprint.iacr.org/2006/043.pdf

Ran Canetti, "The HMAC construction: A decade later," 2007

URI:

http://people.csail.mit.edu/canetti/materials/hmac$10 . \mathrm{pdf}$

Scott Contini; Yiqun Lisa Yin, "Forgery and Partial KeyRecovery Attacks on HMAC and NMAC using Hash Collisions (Extended Version)," 2006

URI: http://eprint.iacr.org/2006/319.pdf

Pierre-Alain Fouque; Gaëtan Leurent; Phong Q. Nguyen, "Full Key-Recovery Attacks on HMAC/NMAC-MD4 and NMAC-MD5," IACR Crypto 2007

URI:

ftp://ftp.di.ens.fr/pub/users/pnguyen/Crypto07.pdf

Vishal Kher; Yongdae Kim, "Securing Distributed Storage: Challenges, Techniques, and Systems" Workshop On Storage Security And Survivability, Nov. 2005

URI = http://doi.acm.org/10.1145/1103780.1103783

Jongsung Kim; Alex Biryukov; Bart Preneel; and Seokhie Hong, "On the Security of HMAC and NMAC Based on HAVAL, MD4, MD5, SHA-0 and SHA-1”, 2006 URI: http://eprint.iacr.org/2006/187.pdf

Dong Hyeok Lee; You Jin Song; Sung Min Lee; Taek Yong Nam; Jong Su Jang, 2007 "How to Construct a New Encryption Scheme Supporting Range Queries on Encrypted Database," Convergence Information Technology, 2007. International Conference on , vol., no., pp.1402-1407, 21-23 Nov. 2007

URI: http://ieeexplore.ieee.org/stamp/stamp.jsp?arnum ber $=4420452 \&$ isnumber $=4420217$

NIST, March 2002. FIPS Pub 198 HMAC specification. $\mathrm{URI}=$

http://csrc.nist.gov/publications/fips/fips198/fips198a.pdf

Kyriacos Pavlou; Richard Snodgrass, "Forensic Analysis of Database Tampering," ACM Transactions on
Database Systems (TODS), 2008

URI = http://doi.acm.org/10.1145/1412331.1412342

PostgreSQL, October 2009. Server Documentation.

$\mathrm{URI}=$

http://www.postgresql.org/docs/8.4/static/index.html

Yu Sasaki, "A Full Key Recovery Attack on HMAC-

AURORA-512,” 2009

URI: http://eprint.iacr.org/2009/125.pdf

Gopalan Sivathanu; Charles P. Wright; and Erez Zadok, "Ensuring data integrity in storage: techniques and applications,” Workshop On Storage Security And Survivability, Nov. 2005

URI = http://doi.acm.org/10.1145/1103780.1103784 Torres et al. 2006a

Elbaz, R.; Torres, L.; Sassatelli, G.; Guillemin, P.; Bardouillet, M.; Rigaud, J.B., 2006a "How to Add the Integrity Checking Capability to Block Encryption Algorithms," Research in Microelectronics and Electronics 2006, Ph. D. , vol., no., pp.369-372, 0-0 0 URI: http://ieeexplore.ieee.org/stamp/stamp.jsp?arnum ber $=1689972 \&$ isnumber $=35631$

Torres et al. 2006b

Elbaz, R.; Torres, L.; Sassatelli, G.; Guillemin, P.; Bardouillet, M., 2006b "PE-ICE: Parallelized Encryption and Integrity Checking Engine," Design and Diagnostics of Electronic Circuits and systems, 2006 IEEE , vol., no., pp.141-142, 0-0 0

URI: http://ieeexplore.ieee.org/stamp/stamp.jsp?arnum ber $=1649595 \&$ isnumber $=34591$ 